\title{
Evidências Preliminares de Validade e Fidedignidade da Versão Brasileira do Child Drawing Hospital
}

\author{
Isabela Andrade de Oliveira Fernandes ${ }^{1}$, Altemir José Gonçalves Barbosa \\ Universidade Federal de Juiz de Fora, Juiz de Fora-MG, Brasil
}

\section{RESUMO}

Para obter evidências de validade e estimativas de fidedignidade para uma medida projetiva de estresse decorrente de hospitalização, a versão brasileira do Child Drawing: Hospital (CD: H) foi aplicada em uma amostra de crianças e adolescentes hospitalizados $(n=81)$ e de uma escola $(n=76)$. Obtiveram-se índices satisfatórios de concordância interavaliadores $(0,448 \geq 0,804)$ e intra-avaliador $(0,975 \geq 1)$ para as três partes (A - Qualidade de Atributos, B - Presença ou Ausência de Atributos e C - Avaliação Global do Desenho) e o escore total do instrumento, bem como para a consistência interna $(\alpha=0,681)$. Os escores do CD: $H$ foram mais elevados na amostra do hospital. Apesar de predominar baixo nível de estresse nos dois subgrupos, havia mais participantes do hospital com Stress Significativo e mais casos de coping, isto é, enfrentamento, na escola. Assim, foram obtidas evidências de validade e estimativas de fidedignidade iniciais para o CD: H. Não obstante, pesquisas adicionais são recomendadas.

Palavras-chave: avaliação psicológica; hospitalização; pediatria; estresse; child drawing: hospital.

ABSTRACT - Preliminary validity and reliability evidence of the Child Drawing: Hospital - Brazilian version

To obtain validity evidence and reliability estimates for a projective measure of stress resulting from hospitalization, the Child Drawing: Hospital - Brazilian version (CD:H) was applied to a sample of hospitalized children and adolescents $(n=81)$ and a school $(n=76)$. Satisfactory indices of inter-rater concordance $(0.488 \geq 0.804)$ and intra-rater $(0.975 \geq 1)$ were obtained for the three parts (A - Attributes Quality, B - Attributes Presence or Absence, and C - Global Drawing Assessment) and the total score of the instrument, as well as for internal consistency $(\alpha=0.681)$. The CD:H scores were higher in the hospital sample. Although there was a predominance of Low Level stress in both subgroups, there were more hospital participants with Significant Stress and more cases of Coping in the school group. Thus, validity evidence and initial reliability estimates were obtained for CD:H. Nevertheless, further research is recommended.

Keywords: Psychological Evaluation; Hospitalization; Pediatrics; Stress; Child Drawing: Hospital.

RESUMEN - Evidencias preliminares de validez y confiabilidad de la versión brasileña del Child Drawing Hospital Para obtener evidencias de validez y estimaciones de confiabilidad para una medida proyectiva de estrés derivada de hospitalización, la versión brasileña del Child Drawing: Hospital (CD: H) fue aplicada en una muestra de niños y adolescentes hospitalizados $(n=81)$ y de una escuela $(n=76)$. Se obtuvieron índices satisfactorios de concordancia inter-evaluadores $(0,448 \geq 0,804)$ e intra-evaluador $(0,975 \geq 1)$ para las tres partes (A - Calidad de Atributos, B - Presencia o Ausencia de Atributos y C - Evaluación Global del Diseño) y la puntuación total del instrumento, así como para la consistencia interna $(\alpha=0,681)$. Los puntajes del CD: H fueron más elevados en la muestra de hospital. A pesar de predominar bajo nivel de estrés en los dos sub-grupos, había más participantes con estrés significativo en el hospital y más casos de Coping, esto es, enfrentamiento, en la escuela. Así, se obtuvieron evidencias de validez y estimaciones de confiabilidad inicial para el CD: H. No obstante, se recomiendan investigaciones adicionales.

Palabras clave: Evaluación Psicológica; Hospitalización; Pediatría; Estrés; Child Drawing Hospital.

\section{Introdução}

O estresse se relaciona diretamente com a adaptação e com a mudança (Faro \& Pereira, 2013). O termo "stress" foi utilizado pela primeira vez na área da saúde em 1926 por Hans Selye, indicando um conjunto de reações que ele havia observado em pacientes acometidos pelas mais variadas patologias (Selye, 1926). Na década de 1930, Selye definiu estresse como uma síndrome geral de adaptação (Selye, 1956). Quatro décadas depois, em 1973, redefiniu como uma resposta não específica do corpo a qualquer exigência (Selye, 1973). De forma geral, Selye (1956) define estresse em termos de "eustress" e "distress". O primeiro se refere à necessidade de um estilo de vida ativo, enquanto o segundo se relaciona aos níveis excessivos que podem impedir a pessoa de utilizar estratégias de coping. Assim, o estresse é uma resposta orgânica inespecífica frente a uma demanda 
qualquer que venha a alterar o estado de homeostase (Selye, 1973).

Lipp (2000), reconhecidamente uma pesquisadora fundamental da área no Brasil, adota a concepção de estresse baseado na resposta. Ela o define como um conjunto de reações emitido pelo organismo quando exposto a todo tipo de estímulo que gere irritação, medo ou felicidade. A resposta à condição estressante é necessária à sobrevivência da espécie, uma vez que sistemas como luta-fuga e sono-vigília estão associados ao estresse (Selye, 1973). O estresse patológico surge quando faltam aos indivíduos estratégias comportamentais para lidar adequadamente com os eventos estressores (Boaventura \& Araújo, 2012).

Segundo Lazarus e Folkman (1984), o estresse é o resultado de um processo de transição em que a pessoa avalia um evento para determinar se é pessoalmente ameaçador, irrelevante ou benigno. Ocorrem duas avaliações: a avaliação primária é centrada na relevância do evento para o bem-estar pessoal; enquanto a avaliação secundária tem como foco encontrar estratégias de coping (Bossert, 1994). Esse processo sofre influência tanto das características pessoais (crenças sobre si e o mundo) quanto do ambiente (fatores temporais). Assim, o estresse, enquanto resposta, deve ser compreendido como um processo e não como uma reação independente. Dessa forma, o estresse só ocorre quando há percepção de que as experiências estressoras transcendem a capacidade do indivíduo lidar de forma satisfatória com o evento ou, ainda, quando as respostas geradas pelo contato com o estressor extrapolam o nível de competência disponível para lidar com a situação (Lazarus \& Folkman, 1984).

Independentemente do modelo teórico, é possível afirmar, de acordo com Lipp (2000), que reações estressantes podem ser prejudiciais à saúde física, psicológica e social do indivíduo e se manifestam em qualquer fase do desenvolvimento humano. Crianças sob condições de estresse constante ou prolongadas podem ter comprometimento de suas funções fisiológicas, psicológicas e cognitivas (Filgueiras, Fontenelle, Mendonça, FioravantiBastos, Poyares, Oliveira, Saboya, \& Marques, 2012; Björkman, 2014). Nesse sentido, o estresse na infância se equipara ao que acomete os adultos (Lipp \& Lucarelli, 2008). A reação da criança frente a eventos excitantes, irritantes, felizes e/ou amedrontadores exige adaptação por parte dela e inclui mudanças psicológicas, físicas e químicas no seu organismo (Lipp \& Lucarelli, 2008; Broering \& Crepaldi, 2011).

Os sintomas do estresse podem ser classificados em dois grandes grupos: físicos (Lipp \& Lucarelli, 2008) ou externos (Kelsey, 2011); e psicológicos (Lipp \& Lucarelli, 2008) ou subjetivos (Kelsey, 2011). Os sintomas do primeiro grupo são mais facilmente reconhecidos e incluem, por exemplo, redução do apetite, enurese, diarreia, dor de cabeça e náusea. Outros não são tão facilmente identificados e são agrupados no segundo conjunto de sintomas (agressividade, teimosia, medo, ansiedade, choro, desânimo, dificuldades interpessoais, etc.). Há que se destacar que, enquanto processo, a ansiedade é um dos componentes do estresse. Se o estresse é compreendido como uma reação decorrente de um evento crítico, a ansiedade sucede-o, sendo acompanhada por outras alterações emocionais (Commodari, 2010), fisiológicas, entre outros, que compõem o estresse.

Um dos eventos que costuma gerar estresse em crianças é a vivência de uma hospitalização (Skipper, Jr. \& Leonard, 1968; Bossert, 1994; Crepaldi \& Hackbarth, 2002; Broering \& Crepaldi, 2011; Kelsey, 2011; Vasques, Bousso, \& Mesdes-Castillo, 2011), podendo, inclusive, gerar danos significativos ao desenvolvimento dela (Carvalho \& Begnis, 2006; Faquinello, Hiragashi, \& Marcon, 2007; Leite \& Shimo, 2007). Assim, é fundamental ter medidas de estresse adequadas para esse contexto, pois os resultados obtidos com elas podem facilitar a elaboração de intervenções que objetivem minimizar o sofrimento decorrente da hospitalização (Azevedo, 2010). O Child Drawing: Hospital (CD:H) (Clatworthy, Simon \& Tiedman, 1999a) é um dos poucos instrumentos que se propõem a avaliar o estresse associado à hospitalização em crianças com idade escolar. Há, é verdade, outros instrumentos internacionais, como o Child Posttraumatic Stress Reaction Index (CPTS-RI) (Frederick, Pynoos, \& Nader, 1992), o Children's PTSD Inventory (Saigh, 1996), o Child Report of Post-traumatic Symptoms/ Parent Report of Posttraumatic Symptoms CROPS/PROPS (Greenwald \& Rubin, 1999), a Pediatric Emotional Distress Scale (PEDS) (Saylor \& Swenson, 1999) e a Child PTSD Symptom Scale (CPSS) (Foa, Johnson, Feeny, \& Treadwell, 2001) para essa fase do desenvolvimento humano, mas focam especificamente o estresse pós-traumático, não fazem uso do desenho, que é uma atividade bastante lúdica e, portanto, altamente recomendável para crianças, e/ou não são específicos para o contexto hospitalar.

No Brasil, existe a Escala de Stress Infantil (ESI2008) que avalia estresse da criança a partir das reações físicas e psicológicas vivenciadas por ela. Trata-se de uma escala com várias fontes de evidência de validade, sendo inclusive recomendada para uso profissional pelo Sistema de Avaliação de Testes Psicológicos (SATEPSI) (CFP Conselho Federal de Psicologia, 2013). Contudo, possui limitações análogas às dos instrumentos internacionais, ou seja, não é específica para o contexto hospitalar e não possui caráter lúdico. Desenvolvido nos EUA, o CD: H se distingue dessas outras medidas de estresse, que se baseiam em indicadores comportamentais, por adotar uma técnica projetiva e lúdica: o desenho (Clatworthy, Simon, \& Tiedman, 1999a). 
As técnicas projetivas caracterizam-se por apresentarem estímulos pouco estruturados, o que permite uma maior variedade de respostas, foco ampliado nos aspectos qualitativos do desempenho e uma maior interação do psicólogo com o avaliando (Meyer \& Kurtz, 2006; Miguel, 2014). O termo "técnicas projetivas" foi cunhado indicando que o mecanismo de projeção estava subjacente à atividade e que essas técnicas permitiam uma apreciação dinâmica e global do indivíduo, muito utilizada em tratamentos e intervenções (Burgess \& Hartman, 1990). Há muito que essas técnicas vêm sendo equivocadamente associadas exclusivamente à abordagem psicanalítica. Meyer e Kurtz (2006) propuseram substituir o nome "técnicas projetivas" para "técnicas de desempenho" de modo a evitar essa má interpretação. É fato que todos os testes de personalidade podem fornecer dados mais ou menos objetivos (Meyer \& Kurtz, 2006), entretanto há que se cuidar para que independentemente do tipo de instrumento, haja o maior rigor metodológico possível (Anastasi \& Urbina, 2000). O uso de técnicas projetivas para mensurar o status emocional de crianças é comum e acontece há muito tempo (Burgess \& Hartman, 1990). Considerando a avaliação psicológica em contextos hospitalares e mais especificamente na pediatria, há que se salientar a relevância de atividades lúdicas. Sabe-se que o desenho é comumente uma atividade agradável e lúdica para crianças (Di Leo, 1983). Além de ser capaz de promover a continuidade do desenvolvimento infantil e de permitir que a criança desenvolva estratégias para lidar com a internação (Mitre \& Gomes, 2004; Mussa \& Malerbi, 2008), o brincar é uma atividade que pode fornecer informações valiosas sobre o funcionamento psicológico na infância.

Contudo, ser lúdica não é uma condição suficiente para uma medida psicológica. O Conselho Federal de Psicologia (CFP) (2003) estabeleceu uma série de quesitos que um teste psicológico deve possuir para ser usado na prática profissional da área. O CD: $\mathrm{H}$ já possui uma versão traduzida para Portugal (Lima \& Lemos, 2011) e para a Suécia (Wennström, Nasic, Hedelin, \& Bergh, 2011). O instrumento original e suas versões têm apresentado boas evidências de validade e estimativas de fidedignidade como preconizado pelo Conselho Federal de Psicologia. A versão portuguesa, contudo, pretende diferentemente da original, ser uma medida global de bem-estar infantil no hospital. Trata-se de uma medida que inclui um conjunto de testes, sendo o CD: $\mathrm{H}$ um deles. As evidências de validade foram obtidas com uma amostra de 31 crianças e adolescentes entre sete e 12 anos com câncer. No estudo da sua adequação, o CD:H revelou ser um instrumento fidedigno, pois foram encontrados resultados muito elevados no cálculo do acordo entre observadores (Lima \& Lemos, 2011). Já a versão sueca do CD:H objetiva medir estresse e ansiedade, tal como a versão original. A amostra contou com 59 crianças entre cinco e 11 anos e foram obtidas evidências de validade de construto (na comparação entre diferentes grupos, houve diferença significativa entre crianças hospitalizadas e crianças na escola), consistência interna (alfa de Cronbach da Parte $A=0,77$ ) e validade semântica (cálculo do acordo interobservadores: Parte $\mathrm{A}=0,8$ a 0,9; Parte $\mathrm{B}=0,21$ a 0,69; Parte $\mathrm{C}=0,39$ a 0,60; e Escore Total $=0,68$ a 0,84 ) (Wennström et al., 2011). Nas partes B e C, verificaram-se correlações baixas, tanto que os autores salientam a necessidade de aprimorar certas partes do instrumento.

As investigações que têm sido feitas sobre o estresse infantil evidenciam a necessidade de avaliar o estresse nessa fase do curso de vida de modo a contribuir para especificar as intervenções a serem feitas na criança, proporcionando redução desse problema de saúde, evitando aparecimento de doenças e outras dificuldades (Lipp; Arantes; Buriti \& Witzig, 2002). Quando se trata de crianças hospitalizadas, identificar os níveis de estresse é ainda mais importante. Assim, este artigo apresenta uma versão brasileira do $\mathrm{CD}: \mathrm{H}$ e os resultados obtidos ao testar a hipótese de que essa medida possui evidências de validade de construto e fidedignidade.

Espera-se que, ao obter evidências de validade para o CD: H e estimar sua fidedignidade no contexto brasileiro, seja disponibilizada uma medida capaz de favorecer o trabalho dos psicólogos hospitalares e das equipes de saúde em enfermarias pediátricas. Reiterase que a internação é uma fonte potencial de estresse infantil, que, se não for bem "trabalhado", pode, inclusive, prejudicar o trabalho médico e agravar a doença que causou a internação. Ter uma medida de estresse em situação de hospitalização com evidências de validade e fidedigna permitirá à equipe interdisciplinar das enfermarias pediátricas planejar, implantar e avaliar projetos que tenham como objetivo minimizar os estressores do ambiente, contribuindo, desse modo, para a humanização dos ambientes hospitalares, que é uma das metas da política pública brasileira em saúde (Brasil, 2008).

\section{Método}

\section{Tradução e Adaptação do CD: H}

Os procedimentos de tradução e adaptação do CD: H (Clatworthy, Simon, \& Tiedman, 1999b) se basearam em parâmetros internacionais, inclusive descritos pela Organização Mundial de Saúde (American Educational Research Association, American Psychological Association \& National Council on Measurement in Education, 1999; Beaton, Bombardier, Guillemin, \& Ferraz, 2000; 2002). Em síntese, foram adotados os seguintes passos: 1. tradução do instrumento para o idioma-alvo; 2. síntese 
das versões traduzidas; 3 . avaliação da versão sintetizada por especialistas; 4. retrotradução (back-translation); e 5. estudo-piloto.

Inicialmente, quatro tradutores bilíngues efetuaram a tradução do instrumento para o português brasileiro, sendo três deles esclarecidos em relação ao instrumento e um quarto que o desconhecia, resultando em quatro versões independentes do instrumento em português. Em seguida, foi feita a síntese das quatro versões traduzidas, avaliando-se a equivalência semântica, idiomática, experiencial e conceitual para as diferentes traduções de cada item. A versão sintetizada foi submetida à avaliação por juízes especialistas que julgaram a abrangência dos itens e as expressões contidas neles como adequadas para o português. A versão em português do Brasil foi retrotraduzida por dois tradutores bilíngues, sendo um deles juramentado. Ela foi enviada para uma das autoras da versão original do instrumento para que o conteúdo fosse avaliado. Após concordância em relação à adequação da versão, procedeu-se a realização do estudo-piloto do qual participaram três psicólogas de um hospital público de Juiz de Fora, MG. Cada uma das psicólogas aplicou o instrumento em duas crianças, avaliou os desenhos e, em entrevistas, relataram não terem tido dificuldades na aplicação e correção do instrumento.

Além de traduzir e adaptar o CD: $\mathrm{H}$, foi analisada a equivalência do uso de lápis de cor ou giz de cera na produção do desenho de uma pessoa no hospital. Esse procedimento foi adotado pelo fato de, no Brasil, o lápis de cor ser mais comumente utilizado no contexto escolar, bem como pela dificuldade para encontrar giz da cor roxa. Para tanto, o CD: $\mathrm{H}$ foi aplicado em uma amostra de escolares $(N=24)$ recrutados em duas turmas, sendo que uma delas utilizou o giz de cera $(n=11)$ e outra desenhou com lápis de cor $(n=13)$. Não foram encontradas diferenças significativas entre os dois grupos tanto para o total do instrumento $\left(t^{\circ}(24 ; 22)=1,348 ; p=0,191\right)$ quanto para as partes $\mathrm{A}\left(t^{\circ}(24 ; 22)=1,322 ; p=0,200\right)$, B $\left(t^{\circ}(24 ; 22)=0,987 ; p=0,340\right)$ e C $\left(t^{\circ}(24 ; 22)=0,436\right.$; $p=0,667)$. Assim, optou-se por utilizar o lápis de cor.

\section{Participantes}

Esta pesquisa contou com a colaboração de 157 participantes, com idades entre 6 e 13 anos, divididos em duas amostras: hospital $(n=81)$ e escola $(n=76)$. As amostras foram obtidas de modo não probabilístico por conveniência. O grupo da escola foi recrutado em uma única instituição pública e cursava o ensino fundamental. As crianças e os adolescentes do hospital foram recrutados em três instituições, sendo duas públicas e uma privada sem fins lucrativos. Todas as instituições - escola e hospitais - são da Zona da Mata de Minas Gerais. A Tabela 1 apresenta o perfil dos participantes. De modo geral, trata-se de uma amostra equilibrada quanto ao sexo dos participantes $\left(\chi_{0}^{2}(157 ; 1)=0,516 ; p=0,473\right)$, com idade média em anos igual a $9,07(\mathrm{DP}=1,68)$ e que cursava o ensino fundamental. Os participantes do hospital $(M=8,62$; $\mathrm{DP}=1,89)$ são mais novos $\left(t^{\circ}(157 ; 155)=3,66 ; p<0,000\right)$ que os da escola $(M=9,55 ; \mathrm{DP}=1,27)$, todavia não foi encontrada correlação entre os escores totais de CD: H e a idade (Pearson $=-0,113$ ). Também participaram do estudo duas avaliadoras que realizaram a pontuação dos desenhos coletados no hospital. Ambas são psicólogas, mestres em Psicologia e com experiência de atuação em pediatria. A avaliação por juízes ou avaliadores independentes tem sido considerada um indicador de qualidade de testes projetivos (Groth-Marnat, 2009; Kirk \& Vigeland, 2014).

Tabela 1

Caracterização Demográfica das Amostras

\begin{tabular}{|c|c|c|c|c|c|c|}
\hline \multirow{2}{*}{$\begin{array}{c}\text { Variáveis } \\
\text { Sexo }\end{array}$} & \multicolumn{2}{|c|}{ Hospital } & \multicolumn{2}{|c|}{ Escola } & \multicolumn{2}{|c|}{ Total } \\
\hline & $\mathrm{n}$ & $\%$ & $\mathrm{n}$ & $\%$ & $\mathrm{n}$ & $\%$ \\
\hline Feminino & 45 & 55,6 & 38 & 50 & 83 & 52,90 \\
\hline Masculino & 36 & 44,4 & 38 & 50 & 74 & 47,10 \\
\hline \multicolumn{7}{|c|}{ Escolaridade } \\
\hline $1^{\circ}$ ano & 19 & 23,5 & - & - & 19 & 12,1 \\
\hline $2^{\circ}$ ano & 3 & 3,7 & 8 & 10,5 & 11 & 7,0 \\
\hline $3^{\circ}$ ano & 18 & 22,2 & 14 & 18,4 & 32 & 20,4 \\
\hline $4^{\circ}$ ano & 11 & 13,6 & 17 & 22,4 & 28 & 17,8 \\
\hline $5^{\circ}$ ano & 20 & 24,7 & 23 & 30,3 & 43 & 27,4 \\
\hline $6^{\circ}$ ano & 8 & 9,9 & 12 & 15,8 & 20 & 12,7 \\
\hline $7^{\circ}$ ano & 2 & 2,5 & 2 & 2,6 & 4 & 2,5 \\
\hline
\end{tabular}




\section{Instrumento}

Foi utilizada a versão brasileira do Child Drawing: Hospital (CD: H). Trata-se de um instrumento de fácil aplicação. Os materiais necessários para aplicar o CD:H incluem uma folha de papel em branco e oito opções de cores de lápis de cor (vermelho, roxo, azul, verde, amarelo, laranja, preto e marrom). Os desenhos são solicitados individualmente. Pede-se que a criança "desenhe uma pessoa no hospital". A pontuação dos desenhos é realizada com base em critérios agrupados em três partes. A primeira (Parte A) possui 14 itens: posição, ação, largura e tamanho da pessoa, os olhos e expressão facial, cor predominante, número de cores utilizadas, uso do papel, colocação no papel, inclusão e tamanho dos equipamentos do hospital e nível de desenvolvimento. Para cada item dessa seção, é atribuída uma pontuação em uma escala que varia entre um e dez. A Parte B possui oito itens, sendo que, para três deles (omissão, exagero e redução em uma parte do corpo), atribui-se zero - ausência - ou cinco - presença - pontos e, para os demais (distorção, omissão de duas ou mais partes do corpo, transparência, perfil misto e sombreamento), atribui-se zero - ausência do item - ou dez - presença. Na terceira parte (Parte C), efetua-se uma avaliação geral do desenho como um todo - Gestalt -, atribuindo-se uma pontuação entre um e dez. O escore total é obtido a partir da soma das três partes e pode variar entre 15 e 215 pontos. Os escores menores do que 43 indicam níveis muito baixos de estresse (coping), 44-83 indicam níveis baixos, 84-129 indicam níveis significativos, 130167 indicam níveis bastante elevados e escores maiores do que 168 indicam níveis patológicos.

\section{Procedimento}

A coleta de dados foi precedida pelos cuidados éticos necessários, incluindo a aprovação da pesquisa por um comitê de ética em pesquisa com seres humanos da Universidade Federal de Juiz de Fora (CAAE: 20471613.0.0000.5147), obtenção do Termo de Consentimento Livre e Esclarecido, etc. O CD:H foi aplicado individualmente, no leito, para a amostra de crianças hospitalizadas. $\mathrm{Na}$ amostra de crianças na escola, foi aplicado em sala de aula coletivamente com resposta individual. Nos dois casos, não houve controle de tempo para a realização do desenho, mas, em média, as crianças realizaram a atividade em 30 minutos.

\section{Análise de Dados}

A análise dos dados se deu com a finalidade de apresentar as primeiras evidências de validade e fidedignidade da versão brasileira do CD: H. Foram utilizadas provas de estatística inferencial com um nível de significância de $5 \%$ para comparar grupos (hospital e escola, masculino e feminino), analisar a concordância interavaliadores e intra-avaliador e a consistência interna da medida. Para tanto, foram utilizados, respectivamente, o Teste $t$ e o qui-quadrado, a correlação intraclasse e o alfa de Cronbach. Baseando-se em Wennström et al. (2011), considerou-se que escores menores do que 43 indicam níveis muito baixos de estresse (coping), 44-83 indicam níveis baixos, 84-129 indicam níveis significativos, 130167 indicam níveis bastante elevados, e escores maiores do que 168 indicam níveis patológicos. Essa classificação foi utilizada para comparar participantes do hospital ou da escola e dos sexos feminino ou masculino com o qui-quadrado. Não se encontrou correlação entre os escores totais do CD: $\mathrm{H}$ e a idade (Pearson=-0,113). Os desenhos do hospital $(n=81)$ foram pontuados por duas avaliadoras e pela autora de modo independente com objetivo de analisar a concordância interavaliadores. As avaliadoras foram submetidas a um treinamento para correção do CD:H com duração de 3 horas. Para verificar a concordância, intra avaliador, a autora realizou uma segunda pontuação dos desenhos do hospital dois meses após a primeira. Para verificar os níveis de concordância interavaliadores e intra-avaliador foi utilizado o coeficiente de correlação intraclasse.

\section{Resultados}

\section{Fidedignidade}

Ao analisar a fidedignidade da Parte A do CD: H com o alfa de Cronbach, obteve-se um coeficiente igual a 0,681. Constatou-se, também, que excluir itens não elevaria substancialmente a consistência interna dessa parte da medida. Verificou-se, ademais, que as correlações item-total corrigidas variaram entre 0,462 e 0,073, sendo que, de modo geral, os coeficientes de correlação estão próximos de 0,3. A fidedignidade do CD: H também foi avaliada por meio da concordância interavaliadores e intra-avaliador. A Tabela 2 apresenta os coeficientes de correlação intraclasse para as três avaliações. Salienta-se que autora foi denominada Avaliador 1. Os outros dois avaliadores participaram de um treinamento para conhecimento do manual e treinamento com pontuação de três desenhos com duração de duas horas. Vale sinalizar que eles não conheciam o instrumento e este foi o primeiro contato. Observa-se que as correlações tendem a ser fortes para o Total e para a Parte A $(0,804-0,759)$, mas para as partes $\mathrm{B}$ e $\mathrm{C}$, elas tendem a ser fracas ainda que coeficientes de correlação moderada e forte sejam observados. Esses resultados assemelham-se aos obtidos por Wennström et al. (2011). No caso da concordância intra-avaliador, observaram-se correlações intraclasse muito forte e até mesmo perfeitas nas três partes do instrumento (Parte $\mathrm{A}=0,995$; Parte $\mathrm{B}=1,00$; Parte $\mathrm{C}=0,975)$, bem como no score total $(0,995)$. 
Tabela 2

Correlação Intraclasse Interavaliadores

\begin{tabular}{|c|c|c|c|}
\hline CD: $\mathrm{H}$ & $\begin{array}{c}\text { Avaliador } 1 \\
\mathrm{X} \\
\text { Avaliador } 2\end{array}$ & $\begin{array}{c}\text { Avaliador } 1 \\
\text { X } \\
\text { Avaliador } 3\end{array}$ & $\begin{array}{c}\text { Avaliador } 2 \\
\text { X } \\
\text { Avaliador } 3\end{array}$ \\
\hline Total & 0,804 & 0,759 & 0,792 \\
\hline Parte A & 0,794 & 0,764 & 0,768 \\
\hline Parte B & 0,450 & 0,455 & 0,605 \\
\hline Parte C & 0,448 & 0,779 & 0,489 \\
\hline
\end{tabular}

\section{Escores do CD: H e Associações com Variáveis Demográficas}

$\mathrm{Na}$ Tabela 3, encontram-se as médias e desvios padrões dos escores parciais e total do CD: $\mathrm{H}$. Ao considerar a classificação dos níveis de estresse proposta por Wennström et al. (2011), observou-se que predominaram $\left(\chi_{0}^{2}(157 ; 2)=109,006 ; p<0,0001\right)$ participantes com baixo nível de stress $(n=114 ; 72,61 \%)$. Havia, também, casos de estresse significativo $(n=22 ; 14,01 \%)$ e de coping $(n=21 ; 13,38 \%)$. Ao comparar os escores parciais e o total do $\mathrm{CD}: \mathrm{H}$ entre os sexos, não foi observada diferença significativa apenas para a Parte B $\left(t^{\circ}(157 ; 155)=-0,955 ; \quad p=0,341\right)$. Os participantes do sexo masculino apresentaram escores mais elevados nas Partes A (masculino $-m=59,30 ; d p=15,27$.

Tabela 3

Médias e Desuios Padrões dos Escores Parciais e Total do CD: H

\begin{tabular}{|c|c|c|}
\hline $\mathrm{CD}: \mathrm{H}$ & $\mathrm{M}$ & $\mathrm{DP}$ \\
\hline Parte A & 56,06 & 14,62 \\
\hline Parte B & 2,52 & 4,82 \\
\hline Parte C & 4,92 & 2,48 \\
\hline Total & 63,50 & 17,96 \\
\hline
\end{tabular}

\section{Validade Discriminante}

Para analisar a validade discriminante do $\mathrm{CD}: \mathrm{H}$, os escores médios dos participantes do hospital e da escola foram comparados. Observaram-se diferenças significativas entre eles nas três partes do instrumento, bem como no escore total (Tabela 5). Os participantes hospitalizados apresentaram níveis mais elevados de estresse $\left(\chi^{2}(157 ; 2)=6,761 ; p<0,01\right)$, uma vez que há mais integrantes do primeiro subgrupo com estresse significativo e mais participantes da escola na fase de coping (Figura 1).
Feminino $-m=53,18 ; \mathrm{dp}=12,45 . t^{0}(157 ; 155)=-2,668$; $p<0,01)$ e C (masculino - $m=5,77 ; \quad d p=2,24$. Feminino $-m=4,15 ; d p=2,44 \cdot t^{\circ}(157 ; 155)=-4,295$; $p<0,01$ ), bem como no total (masculino $-m=67,97$; $d p=18,45$. Feminino $-m=59,51 ; \quad d p=16,63$. $\left.t^{\circ}(157 ; 155)=-3,025 ; \quad p<0,01\right)$. Os participantes do sexo masculino apresentam, evidentemente, níveis mais elevados de estresse (Tabela 4) mais elevados $\left(\chi_{o}^{2}(157 ; 2)=12,710 ; p<0,01\right)$. $\mathrm{O}$ Total do $\mathrm{CD}$ : H não se correlacionou significativamente $(r(157)=-0,112$; $p(157)=0,162)$ com a idade dos participantes. O mesmo ocorreu com as Partes A $(r(157)=-0,100 ; p=0,213)$, B $(r(157)=-0,065 ; \quad p=0,416)$ e C $(r(157)=-0,097$; $p=0,226)$.

Tabela 4

Níveis de Estresse de Acordo com o Sexo dos Participantes

\begin{tabular}{lccccc}
\hline \multirow{2}{*}{$\begin{array}{l}\text { Nível de } \\
\text { estresse }\end{array}$} & \multicolumn{2}{c}{ Feminino } & & \multicolumn{2}{c}{ Masculino } \\
\cline { 2 - 3 } \cline { 5 - 6 } & $\mathrm{n}$ & $\%$ & & $\mathrm{n}$ & $\%$ \\
\hline Coping & 16 & 19,28 & & 5 & 6,76 \\
Baixo & 62 & 74,70 & & 52 & 70,27 \\
Significativo & 5 & 6,02 & & 17 & 22,97 \\
Total & 83 & 100,00 & & 74 & 100,00 \\
\hline
\end{tabular}

Tabela 5

Comparação dos Escores das Partes e Total do CD: H entre os Participantes da Escola e do Hospital

\begin{tabular}{|c|c|c|c|c|}
\hline & : $\mathrm{H}$ & $M$ & $D P$ & $t^{\circ}$ \\
\hline \multirow{2}{*}{ Parte A } & Escola & 53,50 & 14,34 & \multirow{2}{*}{$-2,153^{* *}$} \\
\hline & Hospital & 58,47 & 14,54 & \\
\hline \multirow{2}{*}{ Parte B } & Escola & 1,25 & 3,27 & \multirow{2}{*}{$-3,336^{*}$} \\
\hline & Hospital & 3,70 & 5,69 & \\
\hline \multirow{2}{*}{ Parte C } & Escola & 4,28 & 2,04 & \multirow{2}{*}{$-3,190^{*}$} \\
\hline & Hospital & 5,51 & 2,71 & \\
\hline \multirow{2}{*}{ Total } & Escola & 59,03 & 16,72 & \multirow{2}{*}{$-3,102^{*}$} \\
\hline & Hospital & 67,68 & 18,17 & \\
\hline
\end{tabular}

Nota. ${ }^{*} p<0,01 ;{ }^{* *} p=0,33$ 


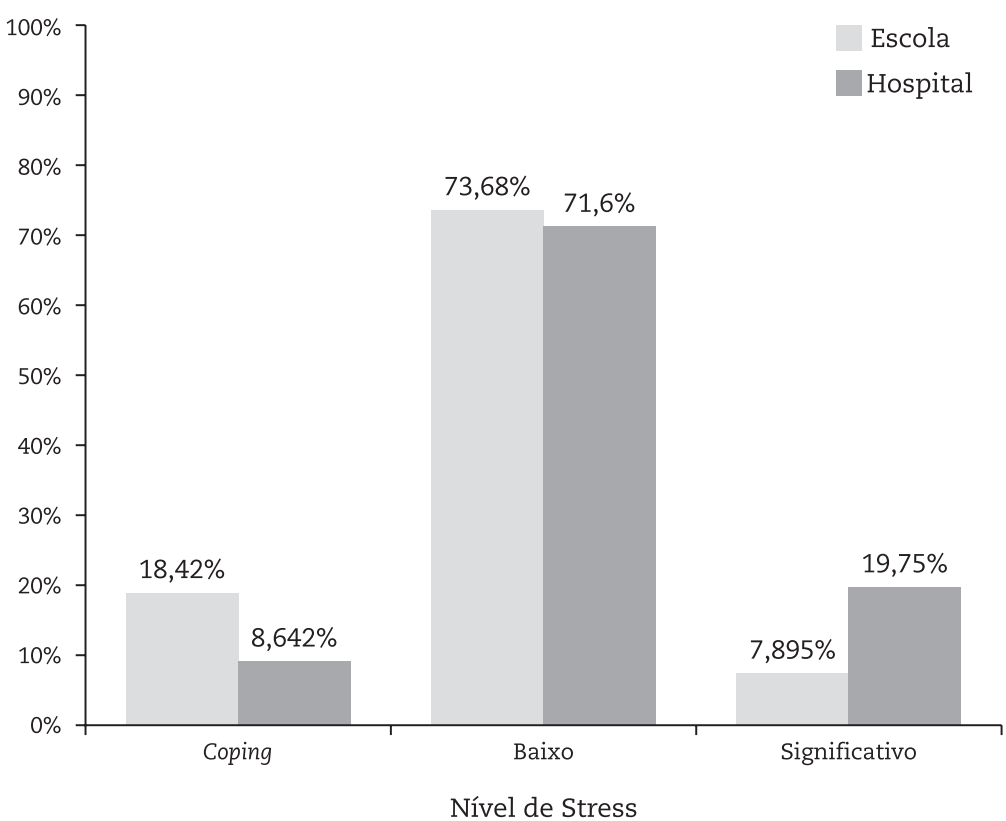

Figura 1. Níveis de stress de participantes do hospital e da escola

\section{Discussão}

Reitera-se que, de modo geral, o estresse pode ser considerado o processo de reação frente a um evento que altere o estado de homeostase em que o indivíduo se encontrava (Selye, 1973; Lazarus \& Folkman, 1984; Lipp, 2000). Assim, a hospitalização pode ser considerada um estressor em potencial para crianças e adolescentes (Skipper, Jr. \& Leonard, 1968; Bossert, 1994; Crepaldi, \& Hackbarth, 2002; Broering \& Crepaldi, 2011; Kelsey, 2011; Vasques, Bousso, \& Mesdes-Castillos, 2011; Boaventura \& Araujo, 2012). Se não for bem enfrentado, o estresse decorrente da hospitalização pode agravar problemas de saúde já existentes ou, até mesmo, gerar outros (Carvalho \& Begnis, 2006; Faquinello, Hiragashi, \& Marcon, 2007; Leite \& Shimo, 2007). Percebe-se, desse modo, a necessidade de uma medida de estresse decorrente da hospitalização para crianças e adolescentes com evidências de validade e fidedignidade. Em se tratando do contexto hospitalar, também é importante que seja um instrumento de fácil e rápida aplicação e que tenha caráter lúdico (Mitre \& Gomes, 2004; Mussa \& Malerbi, 2008). Como o CD: H satisfaz esses requisitos e não possuía uma versão para o Brasil, esta pesquisa traduziu, adaptou e buscou evidências de validade e fidedignidade para a versão brasileira dessa medida. Há que se salientar que desenhos têm se mostrado úteis na avaliação do estado emocional e estresse em crianças e adolescentes, porque estes geralmente se expressam de forma mais clara e aberta ao desenhar do que verbalmente, seja por meio da fala ou da escrita (Meyer \& Kurtz, 2006).
Quanto aos resultados obtidos, o escore médio do Total do CD: H desta pesquisa $(63,5)$ foi inferior ao obtido por Karimi, Fadaiy, Nasrabadi, Godarzi e Mehran (2014), que obtiveram uma média igual a 71,03. Mesmo sendo inferior, neste estudo, predominaram níveis baixos de estresse e não foram identificados casos patológico como no estudo mencionado. Com relação às características demográficas da amostra, uma diferença estatisticamente significativa na pontuação do CD: H entre os sexos foi observada, o que pode indicar um maior nível de estresse em meninos do que nas meninas. Entretanto, são necessários outros estudos para corroborar essa diferença, tendo em vista que os estudos de Karimi et al. (2014), Aminabadi, Ghoreishizadeh, Ghoreishizadeh e Oskouei (2011) e Burns-Nader, Hernandez-Reif e Porter (2014) não encontraram diferenças significativas quando essa variável foi considerada. Em contrapartida, não foram observadas associações significativas entre idade e os escores do CD: H neste estudo. Isso também ocorreu nos estudos de Aminabadi, et al. (2011) e BurnsNader et al. (2014).

Ao analisar as propriedades psicométricas da versão brasileira do CD: H, constatou-se que o nível de consistência interna da Parte A foi semelhante aos resultados obtidos por Wennström et al. (2011) e Karimi et al. (2014). Esses autores obtiveram, respectivamente, alfas Cronbach iguais a 0,77 e 0,74 . Quanto à concordância interavaliadores, os resultados também foram satisfatórios no geral. As correlações intraclasse entre os escores dos avaliadores foram mais elevadas para as Partes A e Total. Nas Partes B e C, os níveis não foram tão elevados, 
mas ainda assim foram significativos. Wennström et al. (2011), Lima e Lemos (2011), Aminabadi et al. (2011), Burns-Nader et al. (2014) e Karimi et al. (2014) também encontraram índices significativos na concordância entre avaliadores. A concordância intra-avaliador foi muito alta. Todavia, esse resultado bastante satisfatório não pode ser profundamente discutido, uma vez que parece que os estudos sobre o CD: H realizados até então não adotaram esse procedimento. Ainda que o resultado tenha sido satisfatório, outra questão relevante é que o fato de as avaliadoras convidadas terem pouco conhecimento sobre o instrumento. Apesar do treinamento, que também teve uma duração limitada, foi a primeira vez que elas pontuaram o CD: H. Isso pode ter diminuído a concordância interavaliadores. Aumentar o tempo de preparação para avaliadores e tornar os critérios de correção ainda mais claros, menos dependentes de treino intensivo, é uma meta a ser atingida em estudos posteriores.

A análise das propriedades psicométricas evidenciou que o CD: $\mathrm{H}$ discriminou níveis de estresse entre um grupo de crianças e adolescentes hospitalizados e um grupo de comparação da escola. Os resultados observados foram muito semelhantes ao que Wennström et al. (2011) e Burns-Nader et al. (2014) encontraram em seus estudos. Foi, obtida, assim, evidência de validade de construto, mais especificamente discriminante, para o instrumento. Ao comparar as pontuações dos dois grupos, percebeu-se que, embora tenham predominado em ambos os grupos participantes com baixo nível de estresse, o grupo do hospital apresentou mais sujeitos com níveis de estresse significativo; enquanto que, no grupo da escola, foram identificados mais casos de coping. Reitera-se que, do mesmo modo que Wennström et al. (2011), não foram encontrados casos de estresse bastante elevado nem níveis patológicos nos dois grupos.

Porém, o estudo de Clathworthy et al. (1999a) com crianças norte-americanas diverge tanto desta pesquisa quanto da investigação sueca já que identificou níveis mais elevados de estresse. O fato de o estudo norte-americano com a versão original do CD: H (Clatworthy et al., 1999a) relatar níveis de estresse mais elevados em comparação a este estudo pode não significar necessariamente que as crianças e adolescentes brasileiros, especialmente os hospitalizados, têm níveis mais baixos de estresse do que os pares estadunidenses. A diferença pode ser decorrente de diferenças culturais, que fazem com que as crianças se expressem de forma diferente em seus desenhos ou as pontuações podem diferir devido a diferenças de interpretação entre avaliadores brasileiros e dos EUA. Além disso, é preciso considerar diferenças entre hospitais, equipes da pediatria, etc. Essas hipóteses precisam ser testadas em outras investigações, incluindo estudos transculturais e transnacionais. Vale reiterar que os estudos realizados na Suécia (Wennström et al., 2011), nos Estados Unidos da América (Burns-Nader et al., 2014) e no Irã (Karimi et al., 2014) também não encontraram casos de estresse patológico, o que pode ser decorrente de limitações do CD: H para identificá-los; algo que também demanda mais pesquisas.

Os resultados discutidos nos parágrafos anteriores devem ser considerados parcimoniosamente devido às limitações deste estudo. Merece menção especial o tamanho da amostra de crianças e adolescentes, e também a de avaliadores. Contar com um número modesto de participantes, ainda que próximo do tamanho das amostras de estudos internacionais (Aminabadi et al., 2011; Wennström, et al., 2011; Lima \& Lemos, 2011; Karimi et al., 2014), diminui a validade externa desta pesquisa. Torna-se fundamental, portanto, realizar estudos com amostras maiores e mais diversificadas. Menciona-se, por exemplo, que não ter nenhum participante hospitalizado em CTI (Centro de Terapia Intensiva) pode ter tido implicações nos escores do CD: $\mathrm{H}$, como no fato de não terem sido identificados níveis mais elevados de estresse. Ainda em relação aos participantes, é sabido que fatores relacionados ao próprio adoecimento (doença crônica, aguda; internações para cirurgia; prognóstico, etc.) e hospitalização (experiência prévia, tempo de internação, alojamento conjunto, etc.) são fatores importantes em relação à forma da criança reagir ao evento estressor e essas variáveis devem ser controladas em estudos posteriores.

Não obstante, sobre as limitações do estudo, é possível afirmar, com base na literatura (Meyer e Kurtz; 2006; Miguel, 2014), que crianças e adolescentes conseguem expressar mais claramente sentimentos, pensamentos e emoções por meio de desenhos. Desse modo, os desenhos são ferramentas de grande valor para "quebrar o gelo" em situações desconhecidas e/ou assustadoras. Eles podem trazer à tona emoções ou pensamentos que as crianças ou adolescentes são incapazes de verbalizar (Meyer \& Kurtz, 2006; Miguel, 2014). As técnicas projetivas apresentam estímulos pouco estruturados, o que garante uma maior amplitude de respostas, maior foco nos aspectos qualitativos do desempenho e uma maior interação do psicólogo com o avaliando (Meyer \& Kurtz, 2006; Miguel, 2014). Em síntese, este estudo permitiu identificar que a versão brasileira do $\mathrm{CD}$ : $\mathrm{H}$ apresenta boa fidedignidade; bem como discrimina entre crianças ou adolescentes hospitalizados e os pares da escola, indicando que o instrumento tem evidências de validade de construto, mais especificamente validade discriminante. No que se refere às implicações práticas, reitera-se que há necessidade de se obter normas brasileiras para, de fato, poder avaliar níveis de estresse de crianças e adolescentes brasileiros hospitalizados. 


\section{Referências}

American Educational Research Association, American Psychological Association \& National Council on Measurement in Education (1999). Standards for Educational and Psychological Testing. New York: American Educational Research Association (AERA).

Aminabadi, N., Ghoreishizadeh, A., Ghoreishizadeh, M., \& Oskouei, S. G. (2011). Can drawing be considered a projective measure for children's distress in paediatric dentistry? International Journal of Paediatric Dentistry, 21, 1-12.

Anastasi, A., \& Urbina, S. (2000). Testagem psicológica. Porto Alegre: Artes Médicas.

Azevedo, A. V. S. (2010). Construção do protocolo de avaliação psicológica hospitalar para a criança queimada. Avaliação Psicológica, 9(1), 99-109.

Beaton, D., Bombardier, C., Guillemin, F., \& Ferraz, M. B. (2000). Guidelines for the process of cross-cultural adaptation of self-report measures. Spine, 25(24), 3186-3191.

Björkman, B. (2014). Children in the radiology department - A study of anxiety, pain, distress and verbal interaction. School of Health Sciences, Jönköping University, Dissertation series $\mathrm{n}^{\circ} 51$.

Boaventura, C. T., \& Araújo, T. C. C. F. (2012). Estresse pós-traumático da criança sobrevivente de câncer e sua percepção acerca da experiência parental. Estudos de Psicologia, 17(2), 283-290.

Bossert, E. (1994). Stress appraisals of hospitalizazed school-age children. Children's health care, 23(1), 33-49.

Brasil. (2008) Ministério da Saúde, Política Nacional de Humanização - HumanizaSUS. Documento de base para gestores e trabalhadores do SUS. Brasília.

Broering, C. V., \& Crepaldi, M. A. (2011). Preparação psicológica e o estresse de crianças submetidas à cirurgias. Psicologia em Estudo (Maringá), $16(1), 15-23$.

Burgess, A. W., \& Hartman, C. R. (1993). Children's drawings. Child Abuse E Neglect, 17(1), 161-168.

Burns-Nader, S., Hernandez-Reif, M., \& Porter, M. (2014). The relationship between mothers' coping patterns and children's anxiety about their hospitalization as reflected in drawings. Journal of Child Health Care, 18(1), 6-18.

Carvalho, A. M., \& Begnis, J. G. (2006). Brincar em unidades de atendimento pediátrico: Aplicações e perspectivas. Psicologia em Estudo Maringá, 11(1), 109-117.

Clatworthy, S., Simon, K., \& Tiedman, M. (1999a). Child drawing: Hospital - An Instrument designed to measure the emotional status of hospitalized school-age children. Journal of pediatric nursing, 14(1), 2-8.

Clatworthy, S., Simon, K., \& Tiedman, M. (1999b). Child drawing: Hospital Manual. Journal of pediatric nursing, 14(1), 10-18.

Commodari, E. (2010). Children staying in hospital: A research on psychological stress of caregivers.Italian Journal of Pediatrics; $36(1) ; 40$.

Conselho Federal de Psicologia. (2003). Resolução n 002/2003, de 24 de março. Brasília, DF: CFP.

Conselho Federal de Psicologia (2013). Sistema de Avaliação de Testes Psicológicos: Testes psicológicos aprovados para uso. Recuperado em 15 de fevereiro, 2013. Recuperado de http://www.pol.org.br/satepsi/sistema/admin.cfm?lista1=sim

Crepaldi, M. A., \& Hackbarth, I. D. (2002). Aspectos psicológicos de crianças hospitalizadas em situação pré-cirúrgica. Temas em Psicologia, 10(2), 99-112.

Di Leo, J. H. (1983). Interpreting Children's Drawings. Brunner/Mizael, Nova Iorque.

Faquinello, P., Higarashi, I., \& Marcon, S. S. (2007). O atendimento humanizado em unidade pediátrica: Percepção de acompanhante da criança hospitalizada. Texto Contexto de Enfermagem, 16(4), 609-616.

Faro, A., \& Pereira, M. E. (2013). Estresse: Revisão narrativa da evolução conceitual, perspectivas teóricas e metodológicas. Psicologia, saúde e doença, 14(1), 78-100.

Filgueiras, Fontenelle, Mendonça, Fioravanti-Bastos, Poyares, Oliveira, Saboya \& Marques (2012). A avaliação do stress em crianças cegas por meio da Escala de Stress Infantil (ESI): diferenças de gênero e de faixas etárias. Rev. Benjamim Constant, 46.

Foa, E., Johnson, K., Feeny, N., \& Treadwell, K. R. (2001). The child PTSD symptom scale: A preliminary examination of its psychometric properties. Journal of Clinical Child Psychology, 30(3), 376-384.

Frederick, C. J., Pynoos, R. S., \& Nader, K. (1992). The child posttraumatic stress disorder reaction index (copyrighted instrument).

Greenwald, R., \& Rubin, A. (1999, January). Brief assessment of children's post-traumatic symptoms: Development and preliminary validation of parent and child scales. Research on Social Work Practice, 9(1), 61-76.

Groth-Marnat, G. (2009). Handbook of psychological assessment. John Wiley \& Sons.

Karimi, R., Fadaiy, Z., Nasrabadi, A. N., Godarzi, Z., \& Mehran, A. (2014). Effectiveness of orientation tour on children's anxiety before elective surgeries. Japan Journal of Nursing Science, 11, 10-15.

Kelsey, S. M. (2011). Stress and coping related to medical illness: Implications for child development (Tese de doutorado não publicada). University of Arizona.

Kirk, C., \& Vigeland, L. (2014). A psychometric review of norm-referenced tests used to assess phonological error patterns. Language, speech, and hearing services in schools, 45(4), 365-377.

Lazarus, R. S., \& Folkman, S. (1984). Stress, appraisal and coping. New York: Springer.

Leite, T. M. C., \& Shimo, A. K. K. (2007). O brinquedo no hospital: Uma análise da produção acadêmica dos enfermeiros brasileiros. Escola Anna Nery Revista de Enfermagem, 11(2), 343-350.

Lima, L., \& Lemos, M. S. (2011). Adequação do child drawing: Hospital na avaliação do bem-estar de crianças hospitalizada portuguesas. Saúde e Qualidade de Vida: uma Meta a Atingir, p. 69-75.

Lipp, M. E. N. (2000) Manual do Inventário de Sintomas de stress para Adultos de Lipp (ISSL). São Paulo: Casa do Psicólogo.

Lipp, M. E. N., Arantes, J. P., Buriti, M. S., \& Witzig, T. (2002). O estresse em escolares. Psicologia Escolar e Educacional, 6(1), 51-56.

Lipp, M. E. N., \& Lucarelli, M. D. M. (2008). Escala de Stress Infantil - ESI: Manual. São Paulo: Casa do Psicólogo.

Meyer, G. J., \& Kurtz, J. E. (2006). Advancing personality assessment terminology: Time to retire "objective" and "projective" as personality test descriptors. Journal of Personality Assessment, 87(3), 223-225.

Mitre, R. M. A., \& Gomes, R. (2004). A promoção do brincar no contexto da hospitalização infantil como ação de saúde. Ciência E Saúde Coletiva, 9(1),147-154. 
Mussa, C., \& Malerbi, F. E. K. (2008). O impacto da atividade lúdica sobre o bem-estar de crianças hospitalizadas. Psicologia: teoria e prática, 10(2), 83-93.

Saigh, P. (1996). The Children's Post Traumatic Stress Disorder-Inventory (CPTSD-I).

Saylor, C. F., \& Swenson, C. C. (1999). The pediatric emotional distress scale: A brief screening measure for young children exposed to traumatic events. Journal of Clinical Child Psychology, 28, 70-81.

Selye, H. (1956). Stress, a tensão da vida. São Paulo: Ibrasa. Edição original publicada por McGraw - Hill Book Company.

Selye, H. (1973). Evolution of the stress concept: The originator of the concept traces its development from the Discovery in 1936 of the alarm reaction to modern therapeutic applications of syntoxic and catatoxic hormones. American Scientist, 61(6), 692-699.

Skipper Jr, J. K., \& Leonard, R. C. (1968). Children, stress and hospitalization: A field experiment. Journal of health and social behavior, 9(4), 275-287.

Vasques, R. C. Y.; Bousso, R. S., \& Mendes-Castillo, A. M. C. (2011). A experiência de sofrimento: Histórias narradas pela criança hospitalizada. Revista da Escola de Enfermagem, 45(1), 122-129.

Wennström, B., Nasic, S., Hedelin, H., \& Bergh, I. (2011). Evaluation of the Swedish version of the Child Drawing: Hospital Manual. Journal of Advanced Nursing, 67(5), 1118-1128.

\section{Sobre os autores}

Isabela Andrade de Oliveira Fernandes é Psicóloga, Mestre em Psicologia pela Universidade Federal de Juiz de Fora e Doutoranda do Programa de Pós-Graduação em Psicologia pela Universidade Federal de Juiz de Fora.

Altemir José Gonçalves Barbosa é Psicólogo, Mestre em Psicologia Escolar e Doutor em Psicologia. Atualmente é docente do Departamento de Psicologia e do Programa de Pós-graduação em Psicologia pela Universidade Federal de Juiz de Fora. 